T. FUJIMAGARI

KŌDAI MATH. SEM. REP.

27 (1976), 11-18

\title{
CONTROLLED GALTON-WATSON PROCESS AND ITS ASYMPTOTIC BEHAVIOR
}

\author{
By Tetsuo FujIMAgaRI
}

1. In a stochastic population process described as a Galton-Watson process each individual splits independently according to a given probability law and new born particles constitute the following generation. In addition to the independence in splitting the law of splitting of each individual depends on neither the generation to which an individual belongs nor the existence of the other individuals of the same generation and is common to all individuals. We shall consider a somewhat generalized Galton-Watson process in the sense that the law of splitting of each individual depends on the total number of individuals of the same generation and the other independence properties are reserved. The object of this note is to study asymptotic behaviors of such processes, although we can hardly obtain any complete results up to now except some partial results. The difficulties in analysing the process will be due to the dependence introduced above from which it no longer holds such as the iteration property of a generating function which plays a fundamental role in GaltonWatson processes.

We shall formulate the process under consideration as follows. Let $Z_{n}$ be the size or the total number of individuals which belong to the $n$-th generation and given a sequence of probability distributions $\mathcal{P}(i)=\left\{p_{r}(i): r \geqq 0\right\}, \imath=0,1,2, \cdots$ where $p_{r}(i) \geqq 0, \sum_{r=0}^{\infty} p_{r}(i)=1$ for all $i \geqq 1$ and $p_{r}(0)=\delta_{r 0}$. Then the process $\left\{Z_{n}\right\}$ may be defined as a Markov chain $\left\{Z_{n}, P_{\imath} ; \imath \in S\right\}$, or briefly $\left\{Z_{n}\right\}$, on the state space $S=\{0,1,2, \cdots\}$ with transition probabilities $P_{\imath \jmath}$ defined by

$$
P_{\imath j}=\sum_{r_{1}+\cdots+r_{i}=j} p_{r_{1}}(i) p_{r_{2}}(i) \cdots p_{r_{i}}(i), \quad P_{0 j}=\delta_{0 j}
$$

for all $\imath, j \geqq 0$.

By the definition of $\left\{Z_{n}\right\}$ each individual belonging to the $n$-th generation splits independently according to the probability law $\mathscr{R}\left(Z_{n}\right)$ and contributes to make the following $(n+1)$-th generation. Since the process $\left\{Z_{n}\right\}$ is the ordinary Galton-Watson process if $\mathscr{P}(1)=\mathscr{P}(2)=\cdots$ and since the law of splitting of an individual is arbitrarily given according to the size of the generation, i. e. $\mathscr{Q}(i)$ is arbitrary for each $i \geqq 1$, we shall call the Markov chain $\left\{Z_{n}, P_{\imath} ; \imath \in S\right\}$ as a controlled Galton-Watson process (CGWP). As seen from the definition the

Received Jan. 30, 1974. 
CGWP $\left\{Z_{n}\right\}$ is one of models which describe branching stochastic processes with interactions between individuals.

Levina, Leontovich, and Pyatetskii-Shapiro [9], and recently Labkovskii [8] considered the same process as a CGWP and obtained a limit theorem concerning to the expectations of certain first exit times of a sequence of CGWP's.

The asymptotic properties of stochastic population processes with interactions between individuals or of "nonlinear stochastic growth models" have been studied by Daley [1], Kesten [3], [4], [5], and Kesten and Stigum [7] etc., and we shall show below that some of results of Kesten and Stigum may be applied to a CGWP under certain conditions.

We shall assume throughout the following condition:

$$
p_{0}(i)+p_{1}(i)<1 \quad \text { for all } i \geqq 1 \text {. }
$$

2. We shall state the following theorem analogous to that of Galton-Watson processes.

THEOREM 1. For each $i \geqq 0$, we have

$$
P_{i}\left(Z_{n} \rightarrow 0 \text { or } \infty \text { as } n \rightarrow \infty\right)=1 \text {. }
$$

Proof. It is sufficient to show that each $i \geqq 1$ is a transient state for $\left\{Z_{n}\right\}$, i. e., $P_{i}\left(Z_{n}=i\right.$ infinitely often $)<1$. First of all, if $p_{0}(i)>0$, then

$$
P_{i}\left(Z_{n}=\imath \text { for some } n \geqq 1\right) \leqq 1-\left(p_{0}(i)\right)^{\imath}<1
$$

and hence $\imath$ is a transient state. We next assume $p_{0}(i)=0$. If $p_{0}(j)=0$ for all $j \geqq 1$, then it is obvious from the assumption (1) that the state $i$ is transient. Thus we assume $J=\left\{j>i: p_{0}(j)>0\right\} \neq \phi$. By denoting the first hitting time to the set $J$ by $\sigma$,

$$
P_{i}\left(Z_{n}=i \text {, i. o. }\right)=P_{i}\left(Z_{n}=i \text {, i. o., } \sigma<\infty\right)+P_{i}\left(Z_{n}=i \text {, i. o., } \sigma=\infty\right),
$$

where we denote "infinitely often" by "i. o.". Since $\sigma=\infty$ implies $Z_{n} \rightarrow \infty$ a. s., the second term of the right-hand side is equal to zero. For the first term we have from the strong Markov property

$$
\begin{aligned}
P_{i}\left(Z_{n}=i, \text { i. o., } \sigma<\infty\right) & =E_{i}\left[\sigma<\infty ; P_{Z_{\sigma}}\left(Z_{n}=i \text {, i. o. }\right)\right] \\
& \leqq E_{i}\left[\sigma<\infty ; 1-\left(p_{0}\left(Z_{\sigma}\right)\right)^{z_{\sigma}}\right] \\
& <1 .
\end{aligned}
$$

Therefore, $P_{i}\left(Z_{n}=i\right.$, i. o. $)<1$, i. e., the state $i$ is transient. The proof is completed.

3. We shall now assume the existence of the first moments $m(i)$ of $\mathscr{P}(i)$, i. e., 


$$
m(i)=\sum_{r=1}^{\infty} r p_{r}(i)<\infty
$$

for all $i \geqq 1$. Then, it is easily seen from the definition of $\left\{Z_{n}\right\}$

$$
E_{i}\left[Z_{n+1} \mid Z_{0}, Z_{1}, \cdots, Z_{n}\right]=E_{\imath}\left[Z_{n+1} \mid Z_{n}\right]=m\left(Z_{n}\right) Z_{n},
$$

and hence if $m(i) \leqq 1$ for all $i \geqq 1,\left\{Z_{n}\right\}$ is a nonnegative supermartingale and converges a.s. to an integrable random variable (see, e.g., Meyer [10]), from which, together with Theorem 1 , we have the following

THEOREM 2. If $m(i) \leqq 1$ for all $i \geqq 1$, then

$$
P_{i}\left(Z_{n} \rightarrow 0 \text { as } n \rightarrow \infty\right)=1 \text { for all } i \geqq 0 \text {. }
$$

Theorem 2 is an analogous statement to the critical or subcritical case of Galton-Watson processes. However even the case of $m(i) \geqq \rho>1$ for all $i \geqq 1$ and for some constant $\rho$ does not imply $q(i)=P_{i}\left(Z_{n} \rightarrow 0\right.$ as $\left.n \rightarrow \infty\right)<1$ for some $i \geqq 1$. This is shown by the following example.

EXAMPLE. Let $\varepsilon$ be a constant such as $0<\varepsilon<1$ and assume $p_{0}(i)=(1-\varepsilon)^{1 / i}$ for all $i \geqq 1$. Moreover we may assume the existence of all the moments of $\mathscr{P}(i)$, $i \geqq 1$ and $m(i) \geqq \rho>1, i \geqq 1$ for some constant $\rho$. Then, $q(i)=1$ for all $i \geqq 0$. For

$$
\begin{aligned}
P_{i}\left(Z_{n} \rightarrow \infty\right) & \leqq P_{i}\left(Z_{1} \geqq 1, Z_{2} \geqq 1, \cdots, Z_{m} \geqq 1\right) \\
& =E_{i}\left[Z_{1} \geqq 1, \cdots, Z_{m-1} \geqq 1 ; P_{Z_{m-1}}\left(Z_{1} \geqq 1\right)\right] \\
& =\varepsilon P_{i}\left(Z_{1} \geqq 1, \cdots, Z_{m-1} \geqq 1\right) \\
& =\cdots \\
& =\varepsilon^{m} \longrightarrow 0 \text { as } m \rightarrow \infty .
\end{aligned}
$$

4. We shall seek for conditions relating to the almost sure extinction of the process $\left\{Z_{n}\right\}$ by means of a comparison method.

Let $h_{i}(s)$ be the generating function of the probability distribution $\mathscr{P}(i)$, i. e., $h_{i}(s)=\sum_{r=0}^{\infty} p_{r}(i) s^{r}, 0 \leqq s \leqq 1$. Moreover, set

$$
\underline{h}(s)=\inf _{i \geqq 0} h_{i}(s), \quad \bar{h}(s)=\sup _{i \geqq 1} h_{i}(s)
$$

and define $\underline{h}^{(n)}(s)$ and $\bar{h}^{(n)}(s)$ for $n \geqq 1$ by the $n$-fold iteration of $\underline{h}(s)$ and $\bar{h}(s)$, respectively. If we put $f_{n}(i ; s)=E_{i}\left(s^{Z_{n}}\right)$, then we have

LEMMA 1 . For all $n \geqq 1$ and $i \geqq 1$,

$$
\left(\underline{h}^{(n)}(s)\right)^{\imath} \leqq f_{n}(i ; s) \leqq\left(\bar{h}^{(n)}(s)\right)^{2}, \quad 0 \leqq s \leqq 1 .
$$

Proof. Since $f_{1}(i ; s)=\left(h_{i}(s)\right)^{2}$, the inequality (3) is obvious for $n=1$. Moreover, since $f_{n}(i ; s)=E_{i}\left[\left(h_{Z_{n-1}}(s)\right)^{z_{n-1}}\right]$, we have 
and hence

$$
E_{i}\left[(\underline{h}(s))^{Z_{n-1}}\right] \leqq f_{n}(i ; s) \leqq E_{i}\left[(\bar{h}(s))^{Z_{n-1}}\right]
$$

$$
f_{n-1}(\imath ; \underline{h}(s)) \leqq f_{n}(i ; s) \leqq f_{n-1}(i ; \bar{h}(s)) .
$$

Therefore, if we assume

$$
\left(\underline{h}^{(n-1)}(s)\right)^{\imath} \leqq f_{n-1}(\imath ; s) \leqq\left(\bar{h}^{(n-1)}(s)\right)^{\imath},
$$

then

$$
\begin{aligned}
\left(\underline{h}^{(n)}(s)\right)^{2} & =\left(\underline{h}^{(n-1)}(\underline{h}(s))\right)^{2} \\
& \leqq f_{n-1}(i ; \underline{h}(s)) \leqq f_{n}(i ; s) \leqq f_{n-1}(i ; \bar{h}(s)) \\
& \leqq\left(\bar{h}^{(n-1)}(\bar{h}(s))\right)^{2}=\left(\bar{h}^{(n)}(s)\right)^{2}
\end{aligned}
$$

and hence the inequality ( 3 ) holds for all $n \geqq 1$ by induction.

LEMMA 2. The limits $\underline{q}=\lim _{n \rightarrow \infty} \underline{h}^{(n)}(0)$ and $\bar{q}=\lim _{n \rightarrow \infty} \bar{h}^{(n)}(0)$ exist and they satisfy the inequality

$$
(\underline{q})^{2} \leqq q(i) \leqq(\bar{q})^{2}, \quad i \geqq 1 .
$$

Proof. Since all $h_{i}(s), i \geqq 1$ are increasing functions in $s, \underline{h}(s)$ and $\bar{h}(s)$ are non-decreasing functions and hence $\underline{h}^{(n)}(s)$ and $\bar{h}^{(n)}(s)$ are also non-decreasing for all $n \geqq 1$. Thus, $\underline{h}^{(n)}(0)=\underline{h}^{(n-1)}(\underline{h}(0)) \geqq \underline{h}^{(n-1)}(0)$ and $\bar{h}^{(n)}(0)=\bar{h}^{(n-1)}(\bar{h}(0)) \geqq \bar{h}^{(n-1)}(0)$, from which the limits $q=\lim _{n \rightarrow \infty} \underline{h}^{(n)}(0)$ and $\bar{q}=\lim _{n \rightarrow \infty} \bar{h}^{(n)}(0)$ exist. Since

$$
\begin{aligned}
\underline{q}(i)=P_{i}\left(Z_{n} \rightarrow 0\right) & =\lim _{n \rightarrow \infty} P_{i}\left(Z_{n}=0\right) \\
& =\lim _{n \rightarrow \infty} f_{n}(i ; 0),
\end{aligned}
$$

we have from Lemma 1 the inequality (4).

LEMMA 3. $\bar{q}$ is a minimal solution of the equation $s=\bar{h}(s)$ such that $0 \leqq s \leqq 1$.

Proof. By the convexity of all functions $h_{i}(s), \bar{h}(s)$ is also a convex function and hence continuous for $0<s<1$. Moreover, the continuity of $\bar{h}(s)$ at $s=0$ and $s=1$ is easily shown by the definition. Thus, from the relation $\bar{h}^{(n)}(0)=\bar{h}\left(\bar{h}^{(n-1)}(0)\right)$, $\bar{q}$ satisfies the equation $s=\bar{h}(s), 0 \leqq s \leqq 1$. If $s_{0}$ is such that $s_{0}=\bar{h}\left(s_{0}\right), 0 \leqq s_{0} \leqq 1$, then $\bar{h}^{(n)}(0) \leqq s_{0}$ for all $n \geqq 1$ and hence $\bar{q} \leqq s_{0}$. This means that $\bar{q}$ is a minimal solution of $s=\bar{h}(s), 0 \leqq s \leqq 1$.

From Lemma 2 and Lemma 3, we have immediately the following theorem.

THEOREM 3. If the equation $s=\bar{h}(s)$ has a solution such that $0 \leqq s<1$, then $q(i)<1$ for all $i \geqq 1$.

REMARK. Since $\underline{h}(s)$ is the infimum of increasing and convex functions $h_{i}(s)$, it is shown that $\underline{h}(s)$ is continuous for $0 \leqq s<1$. Thus, as in the case of $\bar{q}, \underline{q}$ is 
a minimal solution of the equation $s=\underline{h}(s)$ such that $0 \leqq s \leqq 1$. Therefore the sufficient condition $q=1$ for $q(i)=1$ for all $i \geqq 1$ obtained from Lemma 2 is equivalent to that $\underline{h}(s)>s$ for $0 \leqq s<1$. However, since the condition $\underline{h}(s)>s$ for $0 \leqq s<1$ implies $m(i) \leqq 1$ for all $i \geqq 1$, we cannot obtain by this method better sufficient conditions for $q(i)=1$ for all $i \geqq 1$ than Theorem 2 .

From Theorem 3, we have immediately the following corollaries.

COROLLARY 1. If there exist a finite number of generating functions $\varphi_{1}(s)$, $\cdots, \varphi_{k}(s)$ for which each $h_{i}(s)$ is equal to one of them, and if $\varphi_{j}^{\prime}(1)>1$ for all $j=1, \cdots, k$, then $q(i)<1$ for all $i \geqq 1$.

For a cell population process (see Levina, Leontovich, and Pyatetskii-Shapiro [9]), we may assume $p_{0}(i)+p_{2}(i)=1$ for all $i \geqq 1$ and in this case we have:

COROLLARY 2. If $p_{0}(i)+p_{2}(i)=1$ for all $i \geqq 1$ and if there exists a constant $\rho$ such that $m(i) \geqq \rho>1$ for all $i \geqq 1$, then $q(i)<1$ for all $i \geqq 1$.

5. In the case where the laws of splitting $\mathscr{P}(i)$ are the same when the size $i$ is sufficiently large it will be shown that asymptotic behaviors of a CGWP $\left\{Z_{n}\right\}$ are like those of an ordinary Galton-Watson process.

Let $\mathcal{Q}=\left\{p_{r}: r \geqq 0\right\}$ be a probability distribution which satisfies $p_{0}+p_{1}<1$. Assume that there exists an integer $N \geqq 1$ such that $\mathscr{Q}=\mathscr{Q}(i)$ for all $i \geqq N$. For $\mathscr{L}(i), i<N$, it may be taken arbitrarily. Moreover, let $\left\{\tilde{Z}_{n}, \widetilde{P}_{\imath} ; i \in S\right\}$ be a GaltonWatson process with $\mathscr{Q}$ as its splitting law and put $\rho=\sum_{r=1}^{\infty} r p_{r}$ and $q=\tilde{P}_{1}\left(\tilde{Z}_{n} \rightarrow 0\right)$.

LEMMA 4. If $\rho \leqq 1$, then $q(i)=1$ for all $i \geqq 0$.

Proof.

$$
\begin{aligned}
P_{i}\left(Z_{n} \rightarrow \infty\right) & =\lim _{m \rightarrow \infty} P_{i}\left(Z_{n} \rightarrow \infty, Z_{n} \geqq N \text { for all } n \geqq m\right) \\
& =\lim _{m \rightarrow \infty} E_{i}\left[Z_{m} \geqq N ; P_{Z_{m}}\left(Z_{n} \geqq N \text { for all } n \geqq 1, Z_{n} \rightarrow \infty\right)\right]
\end{aligned}
$$

by the Markov property. For $Z_{m} \geqq N$,

$$
\begin{aligned}
& P_{Z_{m}}\left(Z_{n} \geqq N \text { for all } n \geqq 1, Z_{n} \rightarrow \infty\right) \\
= & \widetilde{P}_{Z_{m}}\left(\tilde{Z}_{n} \geqq N \text { for all } n \geqq 1, \tilde{Z}_{n} \rightarrow \infty\right) \\
= & 0,
\end{aligned}
$$

for $\rho \leqq 1$ implies $q^{2}=\tilde{P}_{i}\left(\tilde{Z}_{n} \rightarrow 0\right)=1$ for all $i \geqq 0$. Therefore,

$$
1-q(i)=P_{i}\left(Z_{n} \rightarrow \infty\right)=0, \quad i \geqq 0,
$$

which completes the proof.

LEMMA 5. If $\rho>1$, then $q(i)<1$ for all $i \geqq 1$. 
Proof. First, $q=\tilde{P}_{1}\left(\tilde{Z}_{n} \rightarrow 0\right)<1$ when $\rho>1$ (see, e.g., Harris [2]). For $i \geqq N$,

$$
\begin{aligned}
& P_{i}\left(Z_{n} \geqq i \text { for all } n \geqq 1, Z_{n} \rightarrow \infty\right) \\
= & \widetilde{P}_{i}\left(\tilde{Z}_{n} \geqq i \text { for all } n \geqq 1, \tilde{Z}_{n} \rightarrow \infty\right) \\
\geqq & (1-q)^{\imath}>0,
\end{aligned}
$$

which implies $q(i)=1-P_{i}\left(Z_{n} \rightarrow \infty\right)<1$ for $i \geqq N$. Moreover, from the assumption (1), there exist a finite number of integers $k_{1}, k_{2}, \cdots, k_{m}$ for $1 \leqq i<N$ such that $k_{m} \geqq N$ and

$$
P_{i}\left(Z_{1}=k_{1}, \cdots, Z_{m}=k_{m}, Z_{n} \geqq k_{m} \text { for all } n \geqq m\right)>0 .
$$

Therefore, $q(i)<1$ for $1 \leqq i<N$.

LEMMA 6. If $\rho>1$ and, in addition, $\sum_{r=2}^{\infty}(r \log r) p_{r}<\infty$, then, for each $i \geqq 1$, $\lim _{n \rightarrow \infty} \frac{Z_{n}}{\rho^{n}}=W$ for some $W>0$ holds a.s. $\left(P_{\imath}\right)$ on $\left\{Z_{n} \rightarrow \infty\right\}$.

Proof. Let $i \geqq 1$.

$$
\begin{aligned}
& P_{i}\left(\lim _{n \rightarrow \infty} \frac{Z_{n}}{\rho^{n}}=W \text { for some } W>0, Z_{n} \geqq N \text { for all } n \geqq m\right) \\
= & E_{i}\left[Z_{m} \geqq N ; P_{Z_{m}}\left(Z_{n} \geqq N \text { for all } n \geqq 1, \lim _{n \rightarrow \infty} \frac{Z_{n}}{\rho^{n}}=\widetilde{W} \text { for some } \widetilde{W}>0\right)\right] .
\end{aligned}
$$

For $Z_{m} \geqq N$,

$$
\begin{aligned}
& P_{Z_{m}}\left(Z_{n} \geqq N \text { for all } n \geqq 1, \lim _{n \rightarrow \infty} \frac{Z_{n}}{\rho^{n}}=\widetilde{W} \text { for some } \widetilde{W}>0\right) \\
= & \widetilde{P}_{Z_{m}}\left(\tilde{Z}_{n} \geqq N \text { for all } n \geqq 1, \lim _{n \rightarrow \infty} \frac{\tilde{Z}_{n}}{\rho^{n}}=\widetilde{W} \text { for some } \widetilde{W}>0\right)
\end{aligned}
$$

and since $\rho>1$ and $\sum_{r=2}^{\infty}(r \log r) p_{r}<\infty$ imply

$$
\lim _{n \rightarrow \infty} \frac{\tilde{Z}_{n}}{\rho^{n}}=\widetilde{W} \text { for some } \widetilde{W}>0 \text { a.s. on }\left\{\tilde{Z}_{n} \rightarrow \infty\right\}
$$

(see, Kesten and Stigum [6]), we have

Therefore,

$$
\begin{aligned}
& =\tilde{P}_{z_{m}}\left(\tilde{Z}_{n} \geqq N \text { for all } n \geqq 1\right) \\
& =P_{z_{m}}\left(Z_{n} \geqq N \text { for all } n \geqq 1\right) .
\end{aligned}
$$

$$
\begin{gathered}
P_{i}\left(\lim _{n \rightarrow \infty} \frac{Z_{n}}{\rho_{n}}=W \text { for some } W>0, Z_{n} \geqq N \text { for all } n \geqq m\right) \\
\quad=E_{i}\left[Z_{m} \geqq N ; P_{Z_{m}}\left(Z_{n} \geqq N \text { for all } n \geqq 1\right)\right] \\
=P_{i}\left(Z_{n} \geqq N \text { for all } n \geqq m\right) .
\end{gathered}
$$


By letting $m \rightarrow \infty$, we have

$$
\lim _{n \rightarrow \infty} \frac{Z_{n}}{\rho^{n}}=W \text { for some } W>0 \text { a.s. }\left(P_{\imath}\right) \text { on } \bigcup_{m}\left\{Z_{n} \geqq N \text { for all } n \geqq m\right\}
$$

and since $\left\{Z_{n} \rightarrow \infty\right\} \subset \bigcup_{m}\left\{Z_{n} \geqq N\right.$ for all $\left.n \geqq m\right\}$, we have the lemma.

By Lemmas 4,5 , and 6 above we have immediately the following

THEOREM 4. If there exists an integer $N$ such that $\mathscr{P}=\mathscr{P}(N)=\mathscr{P}(N+1)=\cdots$, then, by putting $\rho=\sum_{r=1}^{\infty} r p_{r}$ for $\mathscr{Q}=\left\{p_{r}: r \geqq 0\right\}$,

(i) $\rho \leqq 1$ implies $q(i)=P_{i}\left(Z_{n} \rightarrow 0\right)=1$ for all $i \geqq 0$ and

(ii) $\rho>1$ implies $P_{i}\left(Z_{n} \rightarrow 0\right)<1$ for all $i \geqq 1$ and the existence of $W=\lim _{n \rightarrow \infty} \frac{Z_{n}}{\rho^{n}}$ a.s. $\left(P_{\imath}\right)$ for all $i \geqq 0$, and moreover, if $\sum_{r=2}^{\infty}(r \log r) p_{r}<\infty$ holds as well as $\rho>1$ we have $P_{i}(W=0)=P_{i}\left(Z_{n} \rightarrow 0\right)$ for all $i \geqq 0$.

The statements of Theorem 4 are well-known properties for ordinary GaltonWatson processes.

REMARK. From the proofs of Lemmas 4,5 and 6 for Theorem 4 we can see that the asymptotic behavior of a CGWP $\left\{Z_{n}\right\}$ does not depend on whatever be the law of splitting when the size of the population is not too large.

6. Finally we state a result obtained by applying the results of Kesten and Stigum (see, Kesten [4] for the results and Kesten and Stigum [7] and Kesten [5] for the proofs) to a CGWP $\left\{Z_{n}\right\}$. It can be shown that the conditions of the theorem of Kesten and Stigum (Kesten [4], Theorem 1 and the following paragraphs) are satisfied for a CGWP $\left\{Z_{n}\right\}$ if there exist constants $K_{1}<\infty$, $K_{2}<\infty, 0<\rho<\infty$, and $\beta>0$ such that it holds

$$
|m(i)-\rho| \leqq K_{1} i^{-\beta} \quad \text { for all } i \geqq 1
$$

and

$$
\sigma^{2}(i)=\sum_{r=0}^{\infty}(r-m(i))^{2} p_{r}(i) \leqq K_{2} \quad \text { for all } i \geqq 1 .
$$

Thus we have the following

THEOREM 5. If the conditions (5) and (6) are satisfied for some constants $K_{1}<\infty, K_{2}<\infty, 0<\rho<\infty$, and $\beta>0$, then we have for $i \geqq 0$

(i) $P_{\imath}\left(\lim _{n \rightarrow \infty} \frac{Z_{n}}{\rho^{n}}=W\right.$ for some $W>0$ or $\left.Z_{n} \rightarrow 0\right)=1$

if $\rho>1$, and

(ii) $P_{i}\left(Z_{n} \rightarrow 0\right)=1$

if $\rho<1$. 
We remark that the conditions for the second statement (ii) of Theorem 5 may be improved by Theorem 2 and the remark following Theorem 4 .

\section{REFERENCES}

[1] D. J. DALEy (1968), Extinction conditions for certain bisexual Galton-Watson branching processes, $Z$. Wahrscheinlichkeitstheorie und Verw. Gebiete 9 315-322.

[2] T. E. HARRIs (1963), The theory of branching processes, Springer.

[3] H. KEsten (1970), Quadratic transformations: A model for population growth, Adv. Appl. Probability, 2, 1-82, 179-228.

[4] H. Kesten (1971), Some nonlinear stochastic growth models, Bull. Amer. Math. Soc., 77, 492-511.

[5] H. Kesten (1972), Limit theorems for stochastic growth models, Adv. Appl. Probability, 4, 193-232, 393-428.

[6] H. Kesten and B.P. STigum (1966), A limit theorem for multidimensional Galton-Watson processes, Ann. Math. Statis. 37, 1211-1223.

[7] H. Kesten and B. P. Stigum (1972), Balanced growth under uncertainty in decomposable economies (preprint).

[8] V.A. LABKovsKII (1972), A limit theorem for generalized branching processes dependent on the size of the population, Teor. Veroyat. Primen., 17, 71-83.

[9] L. V. Levina, A. M. Leontovich and I. I. Pyatetskil-Shapiro (1968), A controllable branching process, Problemy Peredachi Informatsii, 4, 72-82.

[10] P.A. Meyer (1966), Probability and potentials, Blaisdell. 